\title{
Receptivity to free-stream disturbance waves for hypersonic flow over a blunt cone
}

\author{
ZHANG YuDong ${ }^{1,2 \dagger}$, FU DeXun ${ }^{2}$, MA YanWen ${ }^{2}$ \& LI XinLiang ${ }^{2}$ \\ ${ }^{1}$ China Academy of Aerospace Aerodynamics, Beijing 100074, China; \\ ${ }^{2}$ LNM, Institute of Mechanics, Chinese Academy of Sciences, Beijing 100190, China
}

\begin{abstract}
A high-order shock-fitting finite difference scheme is studied and used to do direction numerical simulation (DNS) of hypersonic unsteady flow over a blunt cone with fast acoustic waves in the free stream, and the receptivity problem in the blunt cone hypersonic boundary layers is studied. The results show that the acoustic waves are the strongest disturbance in the blunt cone hypersonic boundary layers. The wave modes of disturbance in the blunt cone boundary layers are first, second, and third modes which are generated and propagated downstream along the wall. The results also show that as the frequency decreases, the amplitudes of wave modes of disturbance increase, but there is a critical value. When frequency is over the critial value, the amplitudes decrease. Because of the discontinuity of curvature along the blunt cone body, the maximum amplitudes as a function of frequencies are not monotone.
\end{abstract}

receptivity, shock-fitting, compact upwind scheme, DNS, blunt cone

Receptivity of hypersonic boundary layers, which is a process of environmental disturbances (including the disturbance in the free stream and the disturbance caused by roughness of body surface), enters the boundary layers, and generates disturbance waves in the boundary layers. Receptivity is the first stage of laminar-turbulent transition. The laminar-turbulent transition process is a result of the nonlinear response of the boundary layers to force disturbances ${ }^{[1]}$. Experimental results show that for supersonic or hypersonic flows, the interaction of the free-stream disturbances with the bow shock affects the stability of the boundary layers and characteristics of laminar-turbulent transition ${ }^{[2]}$. Nevertheless, receptivity is very complex because of the interaction of the free-stream disturbance with the bow shock, and it is very difficult to study the characteristics of disturbance waves in the boundary layers by using linear stability theory. So DNS of receptivity is an important way to study laminar-turbulent transition.

DNS of receptivity of the blunt cone boundary layers requires that the numerical method can

Received February 26, 2007; accepted February 26, 2008; published online September 1, 2008 doi: 10.1007/s11433-008-0164-9

${ }^{\dagger}$ Corresponding author (email: zyd701@sina.com.cn)

Supported by the National Natural Science Foundation of China (Grant Nos. 10632050 and 10502052) 
describe physical quantities on necessary time and space scales, and the numerical error should not pollute the numerical result of the interaction of the free-stream disturbances with the bow shock. The numerical precision of the shock capture method is not enough to simulate the interaction of the free-stream disturbances with the bow shock, so the high order numerical method and the shock-fitting method are needed, in which the bow shock is treated accurately as a boundary. Recently, Zhong et al. $\frac{[2-4]}{}$ studied receptivity of boundary layers of a parabola and a flat plate, but most of aerocrafts' heads are a sphere-cone body, and the bow shock of it is very different from parabola's. There is a high-entropy layer around the head and the curvatures along the sphere-cone body are discontinued. In order to study the effects of the high-entropy layer and discontinued curvature on receptivity of the boundary layers and provide unsteady waves' amplitude and frequency to study laminar-turbulent transition, in this paper, a high-order-accurate shock-fitting scheme is established, based on the fifth-order upwind compact schemes, the sixth-order central schemes and the third-order Runge-Kutta schemes. The receptivity of the hypersonic boundary layers over a blunt cone is simulated.

\section{Numerical methods}

To improve compute precision, based on the fifth-order upwind compact schemes, the sixth-order central schemes and the third-order Runge-Kutta schemes, a high-order-accurate shock-fitting finite difference scheme is established here. To improve compute efficiency, the boundary conditions of the bow shock are established from non-conservation characteristic compatibility equations. The current approach avoids the computation of second derivative of geometric parameters for the shock front. The receptivity of the hypersonic boundary layers of the blunt cone and the interaction of the free-stream disturbance with the bow shock are studied.

\subsection{Governing equations}

The governing equations are solved in the general curvilinear three-dimensional coordinates $(\xi, \eta, \varsigma)$, in which the grid surface $\eta=1$ is body surface, and as the outer boundary, $\eta=\eta_{\max }$ is bow shock. For the blunt cone, the grid surface of $\eta$ is unsteady and the gridlines turn to be shorter or longer with time, but the grid surfaces of $\xi$ and $\varsigma$ are fixed during computation. The Navier-Stokes equations are transformed into the general curvilinear coordinates $(\xi, \eta, \varsigma)$ from Cartesian coordinates $(x, y, z)$ as

$$
\frac{\partial\left(J^{-1} \boldsymbol{U}\right)}{\partial \tau}+\frac{\partial \boldsymbol{E}^{\prime}}{\partial \xi}+\frac{\partial \boldsymbol{F}^{\prime}}{\partial \eta}+\frac{\partial \boldsymbol{G}^{\prime}}{\partial \varsigma}+\frac{\partial \boldsymbol{E}_{v}^{\prime}}{\partial \xi}+\frac{\partial \boldsymbol{F}_{v}^{\prime}}{\partial \eta}+\frac{\partial \boldsymbol{G}_{v}^{\prime}}{\partial \varsigma}=0,
$$

where

$$
\begin{array}{lll}
\boldsymbol{E}^{\prime}=\frac{\boldsymbol{E} \xi_{x}+\boldsymbol{F} \xi_{y}+\boldsymbol{G} \xi_{z}+\boldsymbol{U} \xi_{t}}{J}, & \boldsymbol{F}^{\prime}=\frac{\boldsymbol{E} \eta_{x}+\boldsymbol{F} \eta_{y}+\boldsymbol{G} \eta_{z}+\boldsymbol{U} \eta_{t}}{J}, & \boldsymbol{G}^{\prime}=\frac{\boldsymbol{E}_{\varsigma_{x}}+\boldsymbol{F} \varsigma_{y}+\boldsymbol{G} \varsigma_{z}+\boldsymbol{U} \varsigma_{t}}{J}, \\
\boldsymbol{E}_{v}^{\prime}=\frac{\boldsymbol{E}_{v} \xi_{x}+\boldsymbol{F}_{v} \xi_{y}+\boldsymbol{G}_{v} \xi_{z}}{J}, & \boldsymbol{F}_{\mathbf{v}}^{\prime}=\frac{\boldsymbol{E}_{v} \eta_{x}+\boldsymbol{F}_{v} \eta_{y}+\boldsymbol{G}_{v} \eta_{z}}{J}, & \boldsymbol{G}_{v}^{\prime}=\frac{\boldsymbol{E}_{v} \varsigma_{x}+\boldsymbol{F}_{v} \varsigma_{y}+\boldsymbol{G}_{v} \varsigma_{z}}{J} .
\end{array}
$$

The unsteady flow is simulated by using the moving grid method, in which $\eta_{t}$ and $\partial\left(J^{-1}\right) / \partial t$ are not zero. 


\subsection{Shock-fitting equations}

The flow variables across the shock are governed by shock jump conditions as

$$
\begin{aligned}
& \rho_{1}\left(\boldsymbol{v}_{1} \cdot \boldsymbol{n}-z\right)=\rho_{2}\left(\boldsymbol{v}_{2} \cdot \boldsymbol{n}-z\right), \\
& p_{1} \boldsymbol{n}+\rho_{1} \boldsymbol{v}_{1}\left(\boldsymbol{v}_{1} \cdot \boldsymbol{n}-z\right)=p_{2} \boldsymbol{n}+\rho_{2} \boldsymbol{v}_{2}\left(\boldsymbol{v}_{2} \cdot \boldsymbol{n}-z\right), \\
& \rho_{1} E_{1}\left(\boldsymbol{v}_{1} \cdot \boldsymbol{n}-z\right)+p_{1} \boldsymbol{v}_{1} \cdot \boldsymbol{n}=\rho_{2} E_{2}\left(\boldsymbol{v}_{2} \cdot \boldsymbol{n}-z\right)+p_{2} \boldsymbol{v}_{2} \cdot \boldsymbol{n},
\end{aligned}
$$

where $p$ is pressure, $\rho$ is density, $\boldsymbol{v}$ is the velocity vector, $z$ is the normal velocity of shock, $\boldsymbol{n}$ is the normal vector of shock, subscript 2 represents the variable immediately behind the shock and subscript 1 represents the variables on the free stream side of the shock surface.

On the boundary of the bow shock, the left characteristic compatibility equation along $\eta$ direction is needed here.

The Jacobian matrix along $\eta$ direction is as $\boldsymbol{B}=\frac{\partial \boldsymbol{E}}{\partial \boldsymbol{U}} \eta_{x}+\frac{\partial \boldsymbol{F}}{\partial \boldsymbol{U}} \eta_{y}+\frac{\partial \boldsymbol{G}}{\partial \boldsymbol{U}} \eta_{z}+\eta_{t}$, and its eigenvalue includes

$$
\begin{aligned}
& \left(u \eta_{x}+v \eta_{y}+w \eta_{z}+\eta_{t}\right), \cdots,\left(u \eta_{x}+v \eta_{y}+w \eta_{z}+\eta_{t}\right), \\
& \left(u \eta_{x}+v \eta_{y}+w \eta_{z}+\eta_{t}-c \sqrt{\eta_{x}^{2}+\eta_{y}{ }^{2}+\eta_{z}^{2}}\right), \\
& \left(u \eta_{x}+v \eta_{y}+w \eta_{z}+\eta_{t}+c \sqrt{\eta_{x}{ }^{2}+\eta_{y}{ }^{2}+\eta_{z}^{2}}\right),
\end{aligned}
$$

where $c$ is the sound speed and the corresponding eigenvector of $u \eta_{x}+v \eta_{y}+w \eta_{z}+\eta_{t}+$ $c \sqrt{\eta_{x}^{2}+\eta_{y}^{2}+\eta_{z}^{2}}$ is

$$
\mathbf{I}_{\mathrm{L}}=\left[\begin{array}{c}
\frac{\gamma-1}{2 c}\left(u^{2}+v^{2}+w^{2}\right) \sqrt{\eta_{x}{ }^{2}+\eta_{y}{ }^{2}+\eta_{z}{ }^{2}}-\left(u \eta_{x}+v \eta_{y}+w \eta_{z}\right) \\
\eta_{x}-\frac{\gamma-1}{c} u \sqrt{\eta_{x}{ }^{2}+\eta_{y}{ }^{2}+\eta_{z}^{2}} \\
\eta_{y}-\frac{\gamma-1}{c} v \sqrt{\eta_{x}{ }^{2}+\eta_{y}{ }^{2}+\eta_{z}^{2}} \\
\eta_{z}-\frac{\gamma-1}{c} w \sqrt{\eta_{x}^{2}+\eta_{y}{ }^{2}+\eta_{z}^{2}} \\
\frac{\gamma-1}{c} \sqrt{\eta_{x}{ }^{2}+\eta_{y}{ }^{2}+\eta_{z}^{2}}
\end{array}\right]^{\mathrm{T}}
$$

The characteristic compatibility relation along the $\eta$ direction is obtained as

$$
\begin{aligned}
\boldsymbol{I}_{\mathrm{L}} \cdot[ & \frac{\partial \boldsymbol{U}}{\partial \tau}+\left(\frac{\partial \boldsymbol{E}}{\partial \boldsymbol{U}} \eta_{x}+\frac{\partial \boldsymbol{F}}{\partial \boldsymbol{U}} \eta_{y}+\frac{\partial \boldsymbol{G}}{\partial \boldsymbol{U}} \eta_{z}+\eta_{t}\right) \frac{\partial \boldsymbol{U}}{\partial \eta}+\frac{\partial \boldsymbol{E}}{\partial \xi} \xi_{x}+\frac{\partial \boldsymbol{F}}{\partial \xi} \xi_{y}+\frac{\partial \boldsymbol{G}}{\partial \xi} \xi_{z}+\frac{\partial \boldsymbol{U}}{\partial \xi} \xi_{t} \\
& +\frac{\partial \boldsymbol{E}}{\partial \varsigma} \boldsymbol{\varsigma}_{x}+\frac{\partial \boldsymbol{F}}{\partial \varsigma} \varsigma_{y}+\frac{\partial \boldsymbol{G}}{\partial \varsigma} \boldsymbol{S}_{z}+\frac{\partial \boldsymbol{U}}{\partial \varsigma} \varsigma_{t}+\frac{\partial \boldsymbol{E}_{v}}{\partial \eta} \eta_{x}+\frac{\partial \boldsymbol{F}_{v}}{\partial \eta} \eta_{y}+\frac{\partial \boldsymbol{G}_{v}}{\partial \eta} \eta_{z} \\
& \left.+\frac{\partial \boldsymbol{E}_{v}}{\partial \xi} \xi_{x}+\frac{\partial \boldsymbol{F}_{v}}{\partial \xi} \xi_{y}+\frac{\partial \mathbf{G}_{v}}{\partial \xi} \xi_{z}+\frac{\partial \boldsymbol{E}_{v}}{\partial \varsigma} \varsigma_{x}+\frac{\partial \boldsymbol{F}_{v}}{\partial \varsigma} \varsigma_{y}+\frac{\partial \boldsymbol{G}_{v}}{\partial \varsigma} \varsigma_{z}\right]=0 .
\end{aligned}
$$

The positions and speeds of the unsteady moving of the bow shock are derived from the shock jump conditions and the characteristic compatibility relation. $\eta_{t}$ and $\partial\left(J^{-1}\right) / \partial t$ are calculated by using the moving grid method, so the unsteady N-S equations are calculated. 
The flow variables are non-dimensionalized by using the free-stream velocity $U_{\infty}$, the radius of the sphere head $d$, the free-stream density $d_{\infty}$, and the free-stream temperature $T_{\infty}$, etc.

The upwind compact schemes, which are established by our workshop, are high-order-accurate with low dissipation. These schemes have been used to DNS study of laminar-turbulent transition $^{[5]}$. In this paper, the fifth-order upwind compact schemes, the sixth-order central schemes and the third-order Runge-Kutta schemes are used in the shock-fitting schemes to do DNS of unsteady flows. The shock-fitting schemes in this paper have been used to study the receptivity to the free-stream disturbance waves in the hypersonic boundary layers of parabola ${ }^{[6]}$, and the results agree well with those in ref. [2].

\section{Numerical results}

\subsection{Steady flows of the blunt cone}

In this paper, the sphere-cone angle of the blunt cone is $10^{\circ}$. Figure 1 shows the blunt cone grids. The free-stream conditions are $M a=15$ and $R e=6026$, and the wall temperature is constant as $T_{\mathrm{w}}=193 \mathrm{~K}$.

In general, the vorticity jump condition across a bow shock according to theorem ${ }^{[7]}$ is as

$$
\omega_{\text {shk }}^{*} / k^{*}=u_{\text {to }}^{*} \frac{\left(1-\rho_{\infty}^{*} / \rho_{\mathrm{s}}^{*}\right)^{2}}{\rho_{\infty}^{*} / \rho_{\mathrm{s}}^{*}} .
$$

Figure 2 shows that the solutions of DNS agree well with the theoretical prediction. It also indicates that our high-order-accurate shock-fitting schemes are effective to simulate the bow shock.

Figure 3 shows that $\rho\left(\mathrm{d} u_{t} / \mathrm{d} y_{n}\right)$ along the grid lines is normal to the blunt cone surface at several grid stations.

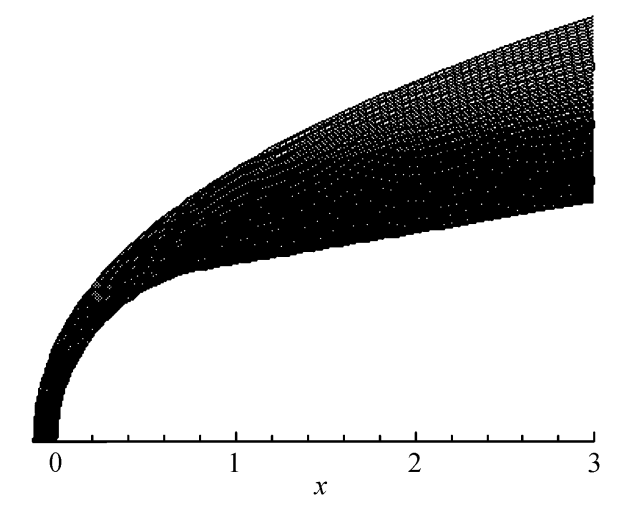

Figure 1 The blunt cone grids.

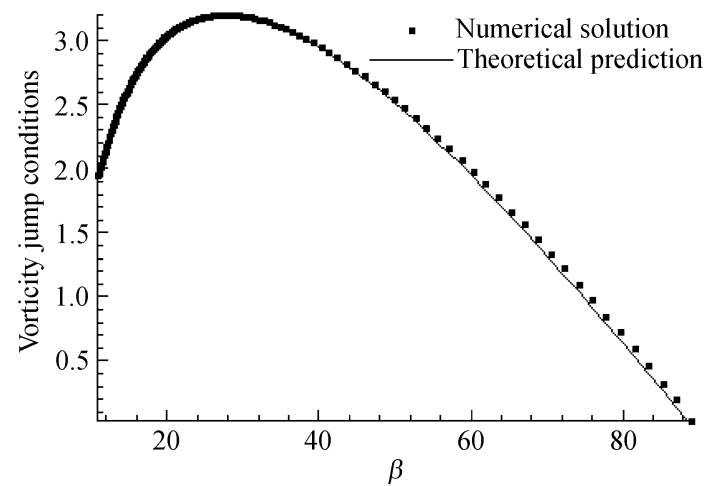

Figure 2 The vorticity jump conditions.

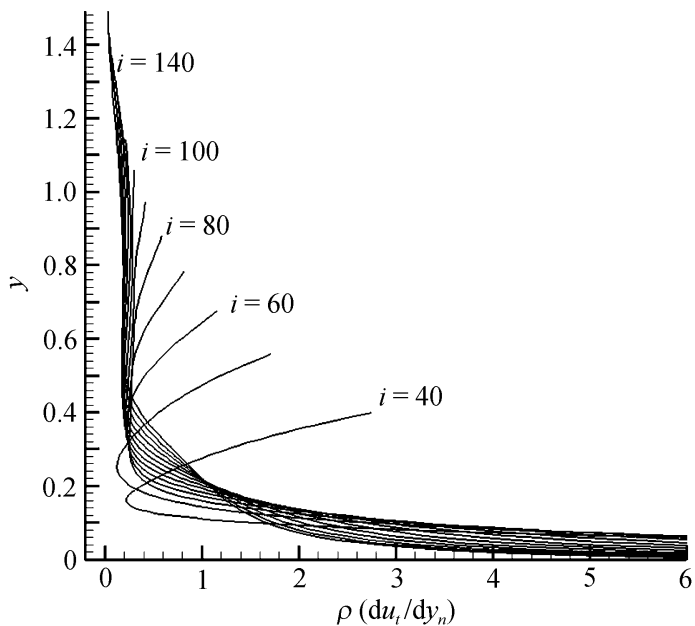

Figure $3 \rho\left(\mathrm{d} u_{t} / \mathrm{d} y_{n}\right)$ along the grid lines normal to the surface $(i=40,60,80,100,140$ corresponds to $x=0.83$, $1.54,2.36,3.42,6.97$, respectively). 
There are generalized inflexions at stations $i=40-50$, which is related with the discontinuity of curvature along the blunt cone surface, and is a necessary condition for the inviscid flow instability.

\subsection{Receptivity characteristic of the blunt cone boundary layers}

The solutions presented in this paper are resolved by $160 \times 120$ grids. The flow conditions are $M a=$ 15 and $R e=6026$, and the wall temperature is constant as $T_{\mathrm{w}}=193 \mathrm{~K}$. The unsteady flow solutions are obtained by imposing weak perturbation waves in a uniform flow in the free stream: fast acoustic waves, slow acoustic waves, entropy waves, and vorticity waves. They are written as

$$
\begin{aligned}
& {\left[\begin{array}{l}
u^{\prime} \\
v^{\prime} \\
p^{\prime} \\
d^{\prime}
\end{array}\right]=\left[\begin{array}{l}
\varepsilon \\
0 \\
\varepsilon / M_{\infty} \\
\varepsilon M_{\infty}
\end{array}\right] \mathrm{e}\left(k x-\frac{F R e}{10^{6}}\right),\left[\begin{array}{l}
u^{\prime} \\
v^{\prime} \\
p^{\prime} \\
d^{\prime}
\end{array}\right]=\left[\begin{array}{l}
\varepsilon \\
0 \\
-\varepsilon / M_{\infty} \\
-\varepsilon M_{\infty}
\end{array}\right] \mathrm{e}^{\mathrm{i}\left(k x-\frac{F R e}{10^{6}} t\right)}, } \\
& {\left[\begin{array}{l}
u^{\prime} \\
v^{\prime} \\
p^{\prime} \\
d^{\prime}
\end{array}\right] }=\left[\begin{array}{l}
0 \\
0 \\
0 \\
\varepsilon M_{\infty}
\end{array}\right] \mathrm{e}^{\mathrm{i}\left(k x-\frac{F R e}{10^{6}} t\right)},\left[\begin{array}{l}
u^{\prime} \\
v^{\prime} \\
p^{\prime} \\
d^{\prime}
\end{array}\right]=\left[\begin{array}{l}
0 \\
\varepsilon \\
0 \\
0
\end{array}\right] \mathrm{e}^{\mathrm{i}\left(k x-\frac{F R e}{10^{6}} t\right),}
\end{aligned}
$$

where $\varepsilon=5 \times 10^{-4}$ is the wave amplitude, $k=15$ is the free-stream wave number, $F=2655$ is the forcing frequency of the free-stream acoustic wave, $x$ is the location and $t$ is the time.

The wave fields are represented by perturbations of instantaneous flow variables with respect to the local steady base flow variables at the same location. $q^{\prime}(x, y, t)=q(x, y, t)-Q(x, y)$, where $q(x, y, t)$ is the instantaneous perturbation obtained by an unsteady numerical simulation of the nonlinear N-S equations, and $Q(x, y)$ is the steady mean flow obtained by a separate steady flow simulation. Figure 4 shows the contours of instantaneous perturbations of velocity along axis $x$, velocity along axis $y$, pressure and vorticity after the flow field reaches a time-periodic state. The instantaneous contours show the interaction of the free-stream disturbances with the bow shock and the development of the disturbance waves in the boundary layer on the surface, and the waves patterns outside the boundary layer and inside the boundary layer are different. The waves in the boundary layer of velocity show that there are three wave modes of disturbance. The wave fields of pressure and vorticity show that acoustic waves behind bow shock enter the boundary layer and interact with the disturbance in the boundary layer, the vorticity waves behind bow shock develop along the body with fluids, and there is a boundary between the disturbance waves outside the boundary layer and inside the boundary layer.

The interaction of any kind of disturbances with a shock wave produces three kinds of waves ${ }^{[8]}$ : acoustic, entropy, and vorticity modes. In this paper, receptivity of the blunt cone boundary layers with different disturbance waves in the free stream is respectively simulated. Figure 5 shows a comparison of pressure, entropy, and vorticity perturbation amplitudes (coming from the Fourier transformation of disturbance variables, see also sec. 2.3) behind the bow shock generated by four kinds of incident waves in the free stream, fast acoustic waves, slow acoustic waves, entropy waves and vorticity waves. The results show that the receptivity in the boundary layers is mainly pressure disturbance, which represents acoustic waves (coordinate scales in Figure 5 are different), vorticity waves' amplitudes are one order smaller than acoustic waves', and entropy waves' are four to five orders smaller than acoustic waves'. In spite of fast acoustic waves, slow acoustic waves or 

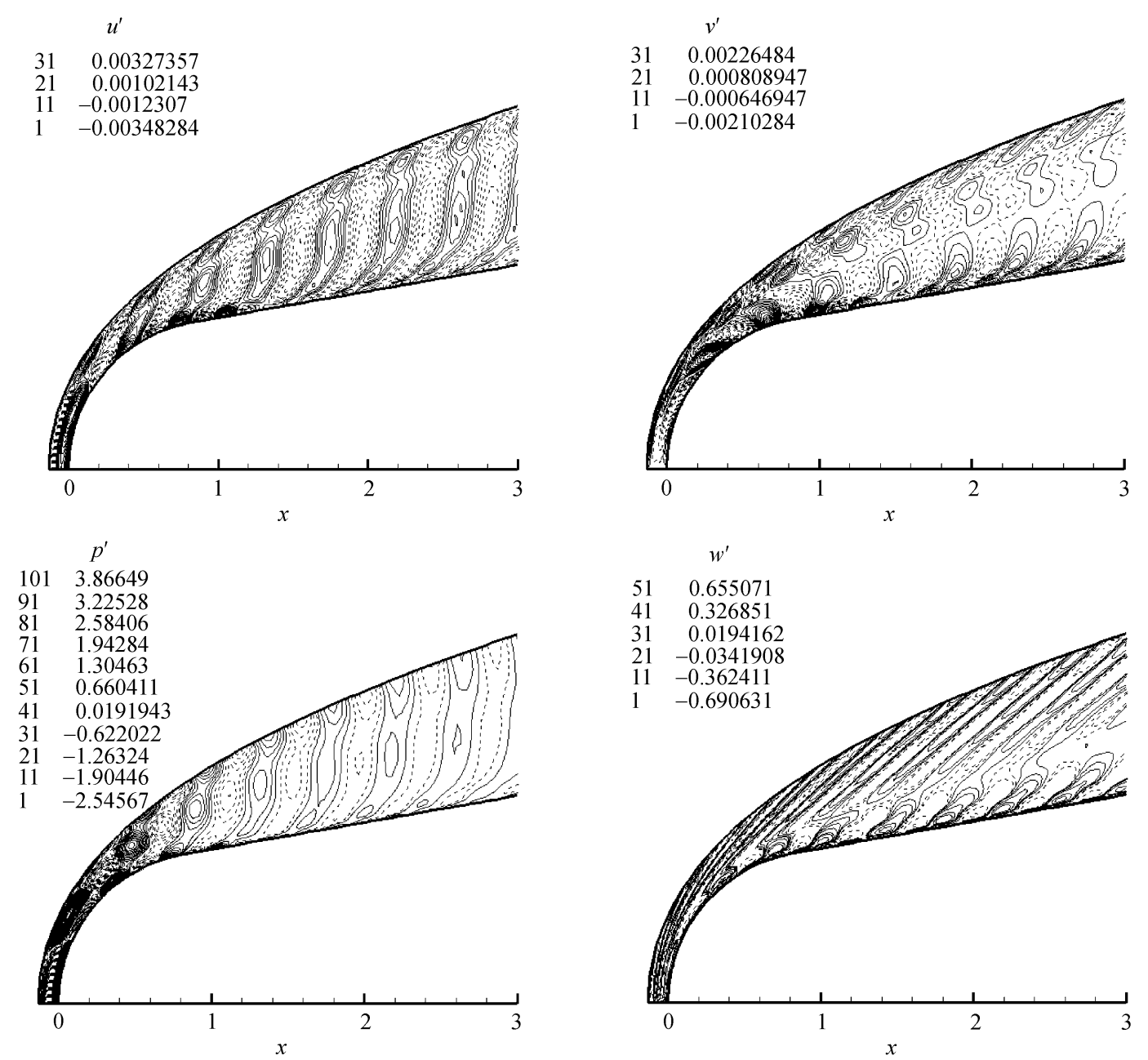

Figure 4 Wave fields of velocity along axis $x$, axis $y$, pressure and vorticity.

entropy waves in a free stream, the amplitudes of acoustic waves, vorticity waves and entropy waves in boundary layers are similar, and are much smaller when vorticity waves are in the free stream. The amplitudes of acoustic waves in the boundary layers from large to small are fast acoustic waves in the free stream, entropy waves in the free stream, slow acoustic waves in the free stream and vorticity waves in the free stream.

\subsection{Wave modes in the blunt cone boundary layers}

In this part, results of DNS of receptivity to the free-stream fast acoustic waves are studied. Temporal Fourier analysis is carried out to study the unsteady disturbance waves mode in the boundary layers, and the real part of Fourier transformation is

$$
q^{\prime}(x, y, t)=\operatorname{Re}\left(\sum_{n=0}^{N}\left|q_{n}^{\prime}(x, y)\right| \mathrm{e}^{\mathrm{i}\left[-n \omega t+\phi_{n}(x, y)\right]}\right) .
$$

The Fourier amplitudes of pressure in the boundary layer for different Fourier frequencies are shown in Figure 6. The amplitude for the fundamental frequency is one order larger than the amplitude for the second or the third harmonic. The results show that waves in the boundary layer are 

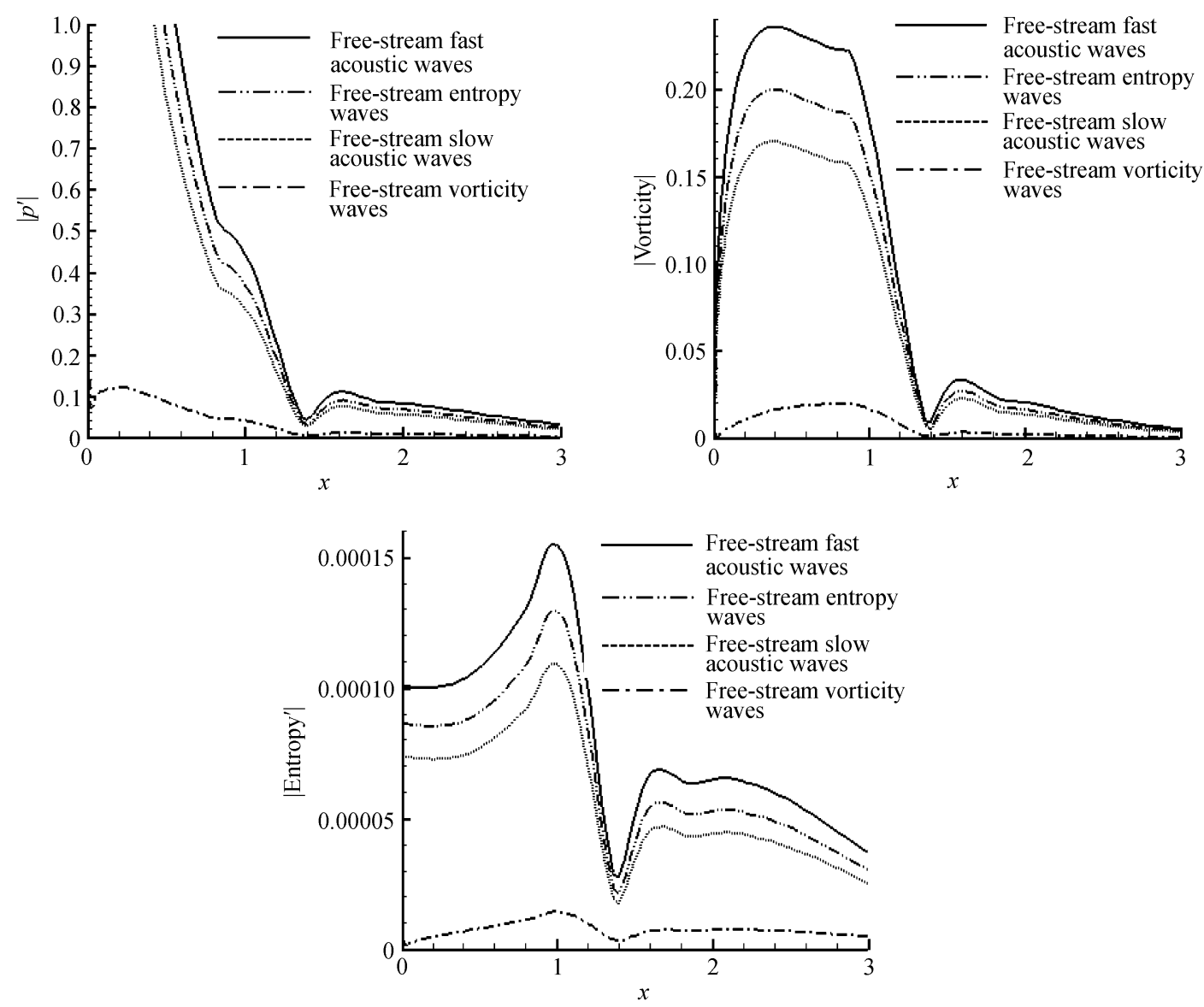

Figure 5 Fourier amplitudes of pressure, entropy, and vorticity in the boundary layers.

mainly a fundamental mode.

To study the unsteady disturbance waves mode in boundary layers, Mack's definitions are used. The number of zeros in the real part of the eigenfunctions of pressure perturbations is used to identify the first, second, and third mode for compressible boundary layers. Figure 7 shows the variation of the real part of the Fourier transform for the pressure perturbations at several grid stations (coordinate scales in Figure 7 are different). As seen, the main wave modes in the blunt cone boundary layers are the first mode near the head and change to the second and third modes at downstream locations.

Figure 8 shows the distribution of the Fourier amplitudes of the velocity perturbations along one grid line near the blunt cone's surface. Figures 4 and 8 show that the main modes of disturbance waves in the boundary layers change around $x=1$.2. Figure 7 shows that it is the first mode before the station, and the second mode after it.

\subsection{Frequency effects}

The parameters of disturbance waves in the free stream affect the results of receptivity. In this part, frequency effects are studied. Figure 9 shows variation of horizontal velocity amplitudes along one grid line near the blunt cone's surface with seven cases of different frequencies. As frequency decreases, the first mode regions extend, and the first mode's decay and the second mode's growth delay in the downstream direction. The amplitudes of disturbance waves in the boundary 

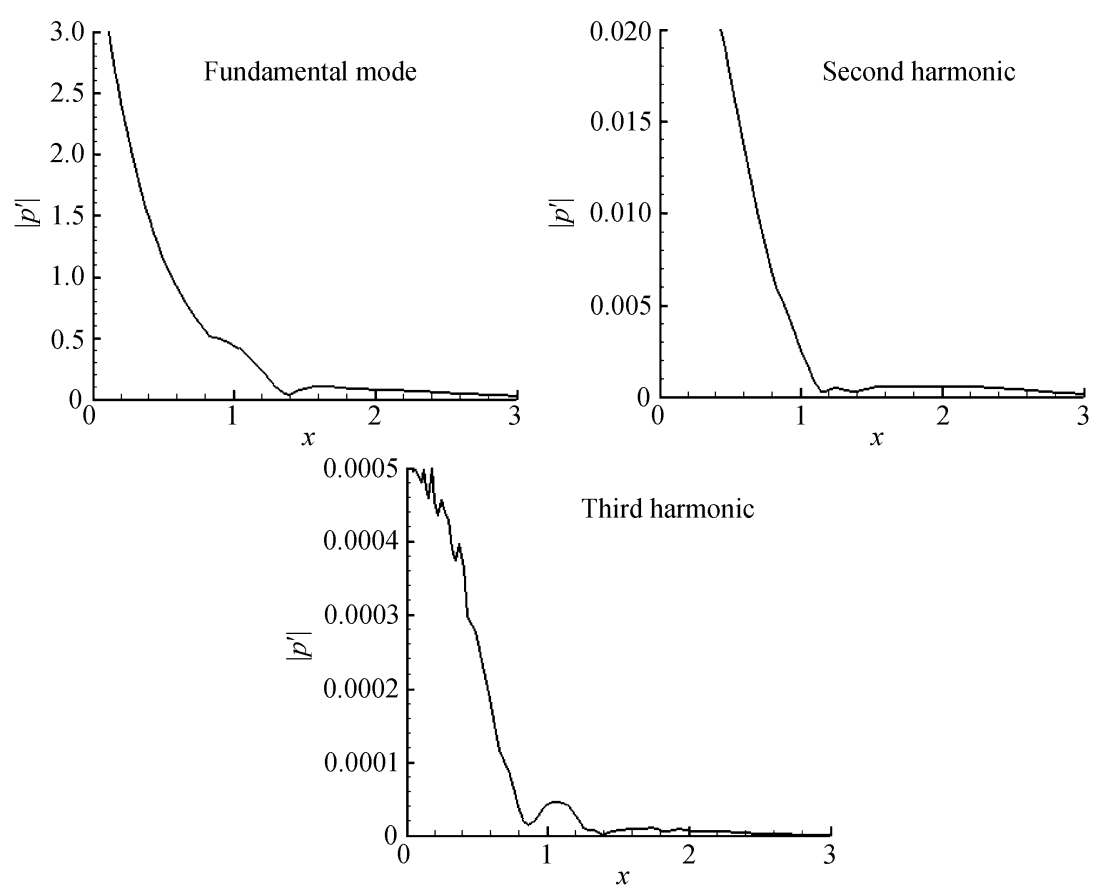

Figure 6 Distribution of amplitudes of pressure perturbations in the boundary layer.
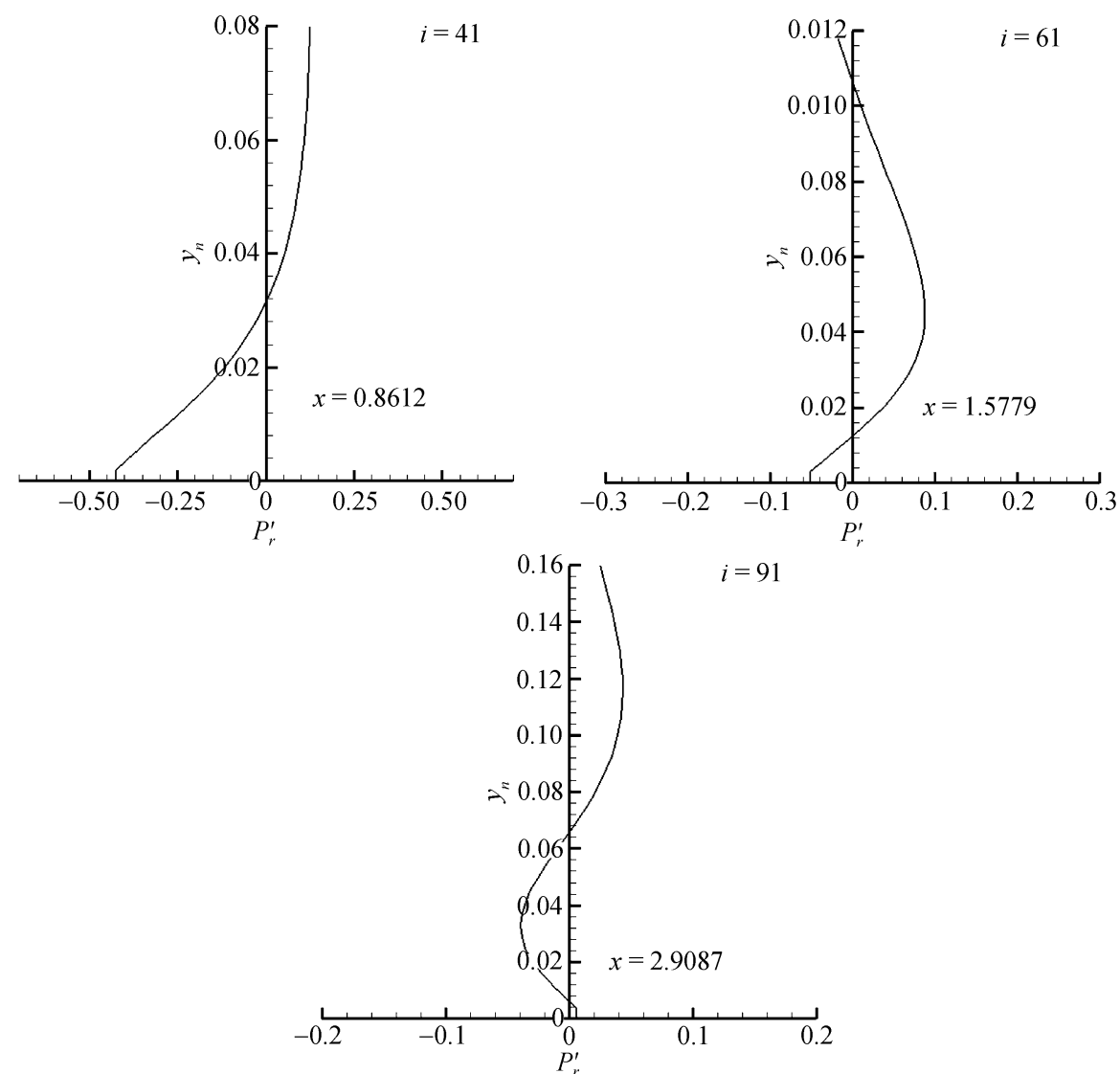

Figure 7 Variation of the real part of the Fourier transform for the pressure perturbations along the grid lines normal to the blunt cone surface at three grid stations. 


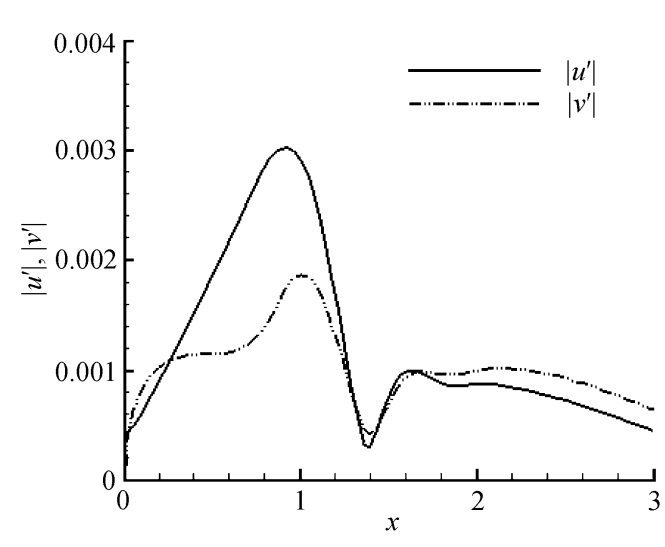

Figure 8 Distribution of the Fourier amplitudes of the velocity perturbations along one grid line near the blunt cone's surface.

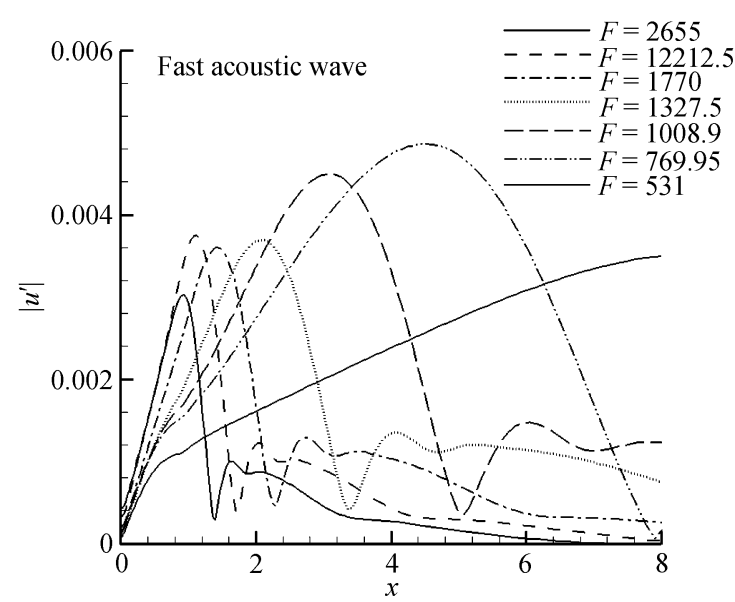

Figure 9 Variation of horizontal velocity amplitudes along one grid line near the blunt cone's surface with seven cases of different frequencies.

layers increase first and then decrease after one critical frequency, because of the discontinuity of curvature along the blunt cone surface, and the maximum horizontal velocity amplitudes as a function of frequencies are not monotone.

\section{Conclusions}

In this paper, a high-order shock-fitting finite difference scheme is established and used to do DNS of the hypersonic unsteady flows of the blunt cone with fast acoustic waves in a free stream. The receptivity to the free stream disturbance waves for the blunt cone hypersonic boundary layers is studied. The results show that:

(1) In spite of any kind disturbance waves in the free stream, the acoustic waves are the strongest disturbance in the blunt cone hypersonic boundary layers.

(2) The main wave modes in the boundary layer are the first mode near the head of the blunt cone and change to the second and third modes at downstream locations.

(3) As frequency decreases, the first mode regions extend, and the first mode's decay and the second mode's growth delay in the downstream direction. The amplitudes of disturbance waves in the boundary layers increase first and then decrease after one critical frequency, because of the discontinuity of curvature along the blunt cone surface, and the maximum horizontal velocity amplitudes as a function of frequencies are not monotone.

1 Reshotko E. Hypersonic stability and transition. In: Desideri J A, Glowinski R, Periaux J, eds. Hypersonic Flows for Reentry Problems, Vol.1. Berlin: Springer, 1991. 18-34

2 Zhong X L. Leading-edge receptivity to free-stream disturbance waves for hypersonic flow over a parabola. J Fluid Mech, 2001, 441: 315-367[DOI]

3 Ma Y B, Zhong X L. Receptivity of a supersonic boundary layer over a flat plate. Part 1: Wave structures and interactions. J Fluid Mech, 2003, 488: 31-78 [DOI]

4 Ma Y B, Zhong X L. Receptivity of a supersonic boundary layer over a flat plate. Part 2: Receptivity to free-stream sound. J Fluid Mech, 2003, 488: 79-121 [DOI]

5 Fu D X, Ma Y W, Liu H. Upwind compact scheme and application. In: Proceedings of the 5th International Symposium on Computational Fluid Dynamics. Nagoya: Japan Society of Computational Fluid Dynamics, 1993. 184-190

6 Zhang Y D, Fu D X, Ma Y W, et al. A high order accurate unsteady shock-fitting method. Chin J Comput Phys, 2007, 24(5): $533-536$

7 Truesdell C. On the curved shocks in steady plane flow of an ideal fluid. J Aero Sci, 1952, 19: 826-828

8 Kovasznay L S G. Turbulence in supersonic flow. J Aero Sci, 1953, 20: 657-682 